\title{
Zuverlässigkeit der Blutgruppenbestimmung bei Neugeborenen
}

\author{
E. A. Bähr, V. Kretschmer \\ Abteilung für Transfusionsmedizin und Gerinnungsphysiologie am Universitätsklinikum der Philipps-Universität Marburg
}

\section{Zusammenfassung:}

Es wurden bei Neugeborenen 2209 Blutgruppenbestimmungen, davon 1121 mal mit Nabelschnurblut und $1088 \mathrm{mal}$ mit Venenblut, mit zwei unterschiedlichen Methoden durchgeführt.

Als "kurze Blutgruppenbestimmung" in Form eines Objektträgertestes mit dem Sediment ungewaschener Erythrozyten und einem Testserensatz Anti-A, $-B,-A B,-D$ und Rh-Kontrollserum ohne Serumgegenprobe und unter Verwendung der Rhesusschaukel für die Rh-Bestimmung.

Als „lange Blutgruppenbestimmung" mit einmal gewaschenen, in physiologischer Kochsalzlösung resuspendierten Erythrozyten und zwei Testserensätzen. Die Antigenaustestung erfolgte auf durchsichtigen Vielfachobjektträgern. Die Serumgegenprobe bestand in einem Dreistufentest im Röhrchen (Kochsalztest $22^{\circ} \mathrm{C}$, Albumintest $37^{\circ} \mathrm{C}$, indirekter Coombstest).

In 11 von 1590 Fällen (0,69\%) ergab die "kurze ABO-Bestimmung” einen falschen Befund. Die Ursache der Fehlbestimmung bestand in unspezifischen Agglutinationen (6 Fälle) und schwachen Reaktionen durch mangelhafte Ausprägung des A-Antigens (5 Fälle). Bei Verwendung von Nabelschnurblut wurde mit Hilfe dieser Methode häufiger einer falsche ABO-Blutgruppe als bei Venenblut gefunden (0,97\% gegenüber $0,18 \%$; $p<0,05)$. Fehlbestimmungen im Rh-Faktor traten in der "kurzen Blutgruppenbestimmung" in 12 von 1590 Fällen (0,75\%) auf. Bei der Austestung des Rh-Faktors wurde häufiger Rh-negativ anstatt Rh-positiv bestimmt (8 Fälle), weil das D-Antigen abgeschwächt reagierte. In vier Fällen wurde Rh-positiv durch unspezifische Agglutinationen vorgetäuscht. Mit Venenblut traten hier häufiger Fehlbestimmungen als mit Nabelschnurblut auf $(1,06 \%$ gegenüber $0,58 \% ; p>0,10)$. Für die $A B O$-Bestimmung erwies sich die Durchführung der Serumgegenprobe als sehr wertvoll. In durchschnittlich 29,3\% der Blutgruppen $A, B$ und 0 waren bereits im Kochsalztest die korrespondierenden Isoagglutinine nachweisbar. Im Albumintest erhöhte sich dieser Anteil auf 32,6\% und im anschließenden indirekten Coombstest auf im Mittel 57,6\%. Dagegen waren irreguläre AB-Antikörper bei den Blutgruppen $A$ und $B$ im Kochsalztest nur in 0,88\%, im Albumintest in 1,1\%, jedoch im indirekten Coombstest immerhin in 11,1\% der Fälle nachweisbar.

Bei Neugeborenen empfiehlt sich daher die Durchführung der "langen ABO-Bestimmung" mit der Serumgegenprobe im Kochsalztest. Der indirekte Coombstest im Rahmen der Serumgegenprobe bleibt der speziellen immunhämatologischen Fragestellung bei O/A-Unverträglichkeit vorbehalten.

Schlüsse/wörter:

Neugeborenenblutgruppe - ABO/Rh-Fehlbestimmung - Nabelschnurblut

\section{Summary:}

2209 blood group determinations, 1121 times using cord blood and 1088, times using venous blood, were performed with two different methods:

$A$ "short blood group determination" as slide test with the pellet of unwashed erythrocytes and a panel of antisera including anti-A, $-B,-A B$, and Rh-serum control without reverse grouping using a Rhesus box for Rhesus testing.

An "extended blood group determination" was performed with erythrocytes washed one time and resuspended in normal saline solution with two different panels of antisera. The determination of the ABOand $R$ h-antigens was done on Bioplates ${ }^{\circledR}$.

Reverse grouping was performed in a three step procedure: saline test $\left(22^{\circ} \mathrm{C}\right)$, albumin test $\left(37^{\circ} \mathrm{C}\right)$ and indirect antiglobulin test.

In 11 out of 1590 cases (0.69\%) a false blood group was determined with the "short ABO typing procedure". Non-specific agglutination (6 cases) and weak agglutination because of an unsufficiently developed antigen A (5 cases) appeared as main reasons for wrong ABO-typing. The frequency of mistyping with this method was higher with cord blood than with venous blood $(0.97 \%$ versus $0.18 \% ; p<0.05)$. Wrong typing of the $R h$-factor was observed in the "short procedure" in 12 out of 1590 cases (0.75\%). Because of weak reaction of the $D$-antigen more often $R h$-negative was determined instead of $R h$-positive (8 cases) than vice versa (4 cases). Non-specific agglutination misled false positive Rh-typing in 4 cases. Mistyping was more frequent with venous blood than with cord blood $(1.06 \%$ versus $0.58 \% ; p>0.10)$. 
Reverse grouping was valuable for the ABO-determination. In $29.3 \%$ of the blood groups $A, B$ and $O$ the compatible alloantibodies were detected with the saline test. With the albumin test the frequency increased to $32.6 \%$ and with the antiglobulin test to $57.6 \%$. Incompatible $A B$-antibodies were found in only $0.88 \%$ of the blood groups $A$ and $B$ with the saline test, in $1.1 \%$ with the albumin test but in $11.1 \%$ with the indirect antiglobulin test.

Blood group typing of newborns should be performed with the "extended blood group determination" which includes reverse grouping with the saline test. Reverse grouping with the indirect antiglobulin test is recommended for the special immunohematological analysis of $A B O$ incompatibility.

Keywords:

Blood group determination of newborns - Frequency of mistyping ABO/Rh - Cord blood

\section{Einleitung}

Es ist bekannt, daß sich bei der Mehrzahl der Neugeborenen agglutinierende $A B$-Antikörper nicht im Blut nachweisen lassen $(1,2)$ oder anderenfalls überwiegend von der Mutter stammen (3-5). Daher ist bei der $\mathrm{Be}$ stimmung der Neugeborenenblutgruppe die alleinige Austestung der Antigene A, B und D üblich (6). Durch unzureichende Antigenausprägung sowie unspezifische Agglutinationen bei Austestung von Nabelschnurblut weisen die ABO-Bestimmungen bei Neugeborenen eine relativ hohe Fehlerquote auf. Wir untersuchten daher die Frage, welche Testfaktoren unter Routinebedingungen zur Steigerung der Zuverlässigkeit der Blutgruppenbestimmung von Neugeborenen beitragen und welche Rolle dabei die Serumgegenprobe einnimmt.

\section{Material und Methoden}

\section{Blutproben}

Es wurde Nativblut von 2209 Neugeborenen, davon $1121 \mathrm{mal}$ Nabelschnurblut und $1088 \mathrm{mal}$ Venenblut, untersucht. In 186 Fällen wurde zusätzlich das mütterliche Blut (Nativblut) herangezogen.

\section{ABO-Rh-Bestimmung}

Zwei verschiedene Methoden wurden routinemäßig parallel eingesetzt:

\section{1. "Kurze Blutgruppe”}

Die Blutgruppenbestimmung wurde als Objektträgertest (Bioplates ${ }^{\circledR}$, Biotest) mit dem Sediment ungewaschener Erythrozyten durchgeführt. Die ABO-Bestimmung erfolgte mit einem Testserensatz Anti-A, $-B$ und $-A B$ (Ortho) mit einer Inkubation von 5 min bei Raumtemperatur. Bei der Rh-Faktorbestimmung wurden die Erythrozyten parallel mit inkomplettem Anti-D und Rh-Kontrollserum (Ortho) für 2 min auf der Rhesusschaukel inkubiert.

\section{2. "Lange Blutgruppe"}

Die Antigenaustestung wurde auf durchsichtigen Vielfachobjektträgern (siehe oben) mit einmal gewaschenen und in physiologischer Kochsalzlösung resuspendierten Erythrozyten durchgeführt. Die ABO-Antigenaustestung erfolgte mit zwei Testserensätzen Anti-A, $-B$ und $-A B$ (Ortho, Biotest) und Inkubation für 20 bis $30 \mathrm{~min}$ bei Raumtemperatur und wurde durch die Serumgegenprobe gegen $A_{1}-, A_{2}-, B$ - und 0 -Zellen als Dreistufentechnik im Röhrchen (Kochsalztest $22^{\circ} \mathrm{C}$, Albumintest $37^{\circ} \mathrm{C}$, indirekter Coombstest) ergänzt. Die Rh-Antigenaustestung erfolgte als Doppelbestimmung mit kompletten Antiseren
Anti-C, -C, -D, -E und -e (Ortho) sowie mit inkompletten Antiseren der gleichen Spezifität (Ortho) unter Zugabe von $22 \%$ igem Rinderalbumin (Ortho) und Inkubation für 20 bis $30 \mathrm{~min}$ bei $37^{\circ} \mathrm{C}$ im Brutschrank.

Für die statistische Auswertung wurde der ChiquadratTest herangezogen.

\section{Ergebnisse}

\section{ABO-Antigenbestimmung}

Bei 11 von insgesamt 1590 (0,69\%) Blutproben Neugeborener wurde $A B O$ anhand der "kurzen Blutgruppe" falsch bestimmt (Tab. 1):

- 5 mal reagierte ein schwaches Antigen (immer A) erst nach dem Waschen und Resuspendieren der Zellen sowie längerer Inkubationsdauer (,lange Blutgruppe”), wobei jedesmal die Serumgegenprobe das passende Antikörpermuster im Kochsalzmilieu erbrachte.

Tab. 1: Fehlbestimmungen der ABO-Blutgruppe bei 1590 Neugeborenenblutproben aufgelistet nach der Ursache der Fehlbestimmung und der Herkunft der Blutproben. (NSB $=$ Nabelschnurblut, $V B=$ Venenblut)

\begin{tabular}{cccc}
\hline Ursache & Gesamt & NSB & VB \\
\hline $\begin{array}{c}\text { Schwaches Antigen } \\
\text { Antigen A }\end{array}$ & 5 & 5 & - \\
B & 5 & 5 & - \\
$\begin{array}{c}\text { Unspezifische Reaktionen } \\
\text { Antigen A }\end{array}$ & 6 & - & - \\
B & 1 & 5 & 1 \\
AB & 4 & 1 & - \\
\hline Gesamt & 1 & 4 & - \\
Blutproben & $11^{\circ}$ & 10 & 1 \\
\hline
\end{tabular}

Tab. 2: Fehlbestimmungen der ABO-Blutgruppe bei 1590 Neugeborenenblutproben aufgelistet nach der Verteilung auf die Blutgruppen $A, B, A B$ und $O$

\begin{tabular}{llllll}
\hline $\begin{array}{l}\text { "Lange } \\
\text { Blutgruppe } \\
\text { (richtig) }\end{array}$ & \multicolumn{5}{c}{ „Kurze Blutgruppe“ (falsch) } \\
\cline { 2 - 6 } & 0 & A & B & AB & Gesamt \\
\hline 0 & - & - & - & 1 & 1 \\
A & 1 & - & - & 4 & 5 \\
B & - & - & - & 1 & 1 \\
AB & - & - & 4 & - & 4 \\
\hline Gesamt & 1 & - & 4 & 6 & 11 \\
\hline
\end{tabular}


- 6mal führten unspezifische Reaktionen zur Diagnose falscher Antigene, wobei besonders das Antigen B zu oft angenommen wurde.

- Als falsch bestimmte ABO-Blutgruppe imponierten $A B$ statt $A$ sowie $B$ statt $A B$ (je 4mal); weiterhin fand sich 2 mal $A B$ statt $O$ bzw. B sowie 1 mal 0 statt $A$ (Tab. 2).

- Mit Venenblut von Neugeborenen ergaben sich seltener Fehlbestimmungen als mit Nabelschnurblut (1 von 526 Blutproben gegenüber 10 von 1028 Blutproben; $p<0,05$ ).

\section{Nachweis von AB-Antikörpern}

Agglutinierende, im Kochsalztest reagierende AB-Antikörper ließen sich passend zum Antigenmuster (nach $A b$ zug der Fälle mit irregulären AB-Antikörpern) in 29,3\% der Blutproben feststellen (Tab. 3). Darüber hinaus wiesen 99 von 100 Blutproben mit Blutgruppe $A B$ keine $A g$ glutinine auf, so daß insgesamt $32,5 \%$ der $A B 0$-Blutgruppen allein anhand der $A B$-Antikörper richtig bestimmbar waren. Weitere $11,3 \%$ der Blutproben mit Blutgruppe 0 wiesen nur Anti-A (6,6\%) oder Anti-B (4,7\%) auf.

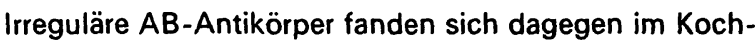
salztest nur selten $(0,5 \%)$. Insgesamt ließen sich agglutinierende und inkomplette $A B$-Antikörper passend zum Antigenmuster (nach Abzug der irregulären $A B$-Antikörper) sogar in $57,3 \%$ der Blutproben mittels Dreistufentest nachweisen. Die 99 von 100 Serumproben von Neugeborenen mit Blutgruppe $A B$ zeigten auch im Albumin und indirekten Coombstest keine $A B$-Antikörper, so daß insgesamt $59,2 \%$ der $A B 0$-Blutgruppenbestimmungen allein anhand der AB-Antikörper mit Hilfe des Dreistufentests sicher bestimmbar waren (Tab. 4).

Andererseits erhöhte sich die Frequenz der irregulären AB-Antikörper bei Durchführung der Serumgegenprobe im Albumin- und indirekten Coombstest auf das 22fache $(11,1 \%)$. Gleichzeitig fand sich bei Blutgruppe 0 in 12,3\% allein Anti-B. Bei Vergleich von Nabelschnur- und Venenblut hinsichtlich der Nachweisbarkeit der AB-Antikörper (Tab. 5) ergab sich bei Blutgruppe $A$ überraschenderweise eine signifikant höhere Frequenz von Anti-B in Nabelschnurblut ( $p<0,01$ ). Für die übrigen Blutgruppen, $B, 0$ und $A B$ konnten einzeln ausgewertet keine signifikanten Unterschiede zwischen Nabelschnur- und Venenblut in der Nachweisfrequenz kompatibler AB-Antikörper gefunden werden $(B: p \gg 0,10 ; 0: 0,10>p$ $>0,05 ; A B: p \gg 0,10)$. Wenn man jedoch die $A B O$ Antikörper bei allen vier Blutgruppen (A, B, $A B, 0)$ zusammen betrachtete, ergab sich eine deutlich höhere Fre-

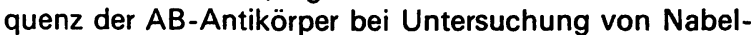
schnurblut $(p<0,01)$.

Die Nachweisbarkeit von AB-Antikörpern im Nabelschnurblut korrelierte eindeutig mit der Blutgruppe der Mutter (Abb. 1). Hatte die Mutter die Blutgruppè 0 , so fanden sich beim Kind wesentlich häufiger AB-Antikörper als bei Blutgruppe $A$. Dennoch ließen sich im kindlichen Blut fast nur die zum Antigenmuster des Kindes passenden $A B$-Antikörper anhand des Kochsalztests nachweisen (siehe auch oben).

\section{Rhesusfaktorfehlbestimmungen}

Bei $0,75 \%$ der Blutproben Neugeborener wurde der RhFaktor anhand der "kurzen Blutgruppe" fehlbestimmt (Tab. 6):

- 5 mal ergab ein schwach ausgeprägtes D-Merkmal eine negative Reaktion. Darüber hinaus wurde $3 \mathrm{mal}$ die sehr
Tab. 3: Häufigkeit von agglutinierenden AB-Antikörpern im Kochsalztest ("reguläre" Antikörper) gegenüber nicht zur Blutgruppe des Neugeborenen passenden AB-Antikörper (.,irreguläre" Antikörper) bel 2189 untersuchten Blutproben

\begin{tabular}{|c|c|c|c|c|}
\hline & \multirow[t]{2}{*}{$\mathbf{N}$} & & \multicolumn{2}{|c|}{ AB-Antikörper } \\
\hline & & & „Reguläre” & .Irreguläre" \\
\hline A & 971 & & $235(24,2 \%)$ & $8(0,8 \%)$ \\
\hline B & 272 & & $88(32,4 \%)$ & $3(1,1 \%)$ \\
\hline \multirow[t]{2}{*}{0} & \multirow[t]{2}{*}{846} & \multirow{2}{*}{$\begin{array}{l}\text { Anti-A,B } \\
\text { allein } \\
\text { Anti-A } \\
\text { Anti-B }\end{array}$} & $289(34,2 \%)$ & \\
\hline & & & $\begin{array}{ll}56 & (6,6 \%) \\
40 & (4,7 \%)\end{array}$ & \\
\hline \multicolumn{2}{|c|}{ Gesamt 2089} & & $612(29.3 \%)$ & $11(0,5 \%)$ \\
\hline$A B$ & 100 & &.- & $1(1,0 \%)$ \\
\hline
\end{tabular}

Tab. 4: Häufigkeit von kompatiblen agglutinierenden und in kompletten AB-Antikörpern im Dreistufentest (",reguläre" Antikörper) gegenüber nicht zur Blutgruppe des Neugeborenen passenden AB-Antikörpern („irreguläre" Antikörper) bei 2189 untersuchten Blutproben

\begin{tabular}{|c|c|c|c|c|}
\hline & \multirow[t]{2}{*}{$\mathbf{N}$} & & \multicolumn{2}{|l|}{ AB-Antikörper } \\
\hline & & & „Reguläre“ & .Irreguläre" \\
\hline A & 971 & . & $520(53,6 \%)$ & $117(12,2 \%)$ \\
\hline B & 272 & & $173(63,6 \%)$ & $21 \quad(7,7 \%)$ \\
\hline 0 & 846 & $\begin{array}{l}\text { Anti-A,B } \\
\text { allein } \\
\text { Anti-A } \\
\text { Anti-B }\end{array}$ & $\begin{array}{r}504(59,6 \%) \\
50(5,9 \%) \\
104(12,3 \%)\end{array}$ & \\
\hline \multicolumn{2}{|c|}{ Gesamt 2089} & & $1197(57,3 \%)$ & $138(11,1 \%)$ \\
\hline$A B$ & 100 & & & $1 \quad(1,0 \%)$ \\
\hline
\end{tabular}

Tab. 5: Häufigkeit von „regulären” agglutinierenden AB-Antikörpern im Kochsalz in Nabelschnur- und Venenblut. (NSB $=$ Nabelschnurblut, $V B=$ Veneriblut)

\begin{tabular}{lccccc}
\hline & NSB & & \multicolumn{2}{l}{ VB } \\
\cline { 2 - 3 } \cline { 6 - 7 } & Gesamt & N & & Gesamt & N \\
\hline A & 491 & $139(28,3 \%)$ & & 480 & $96(20,0 \%)$ \\
B & 146 & $47(32,2 \%)$ & 126 & $41(32,5 \%)$ \\
0 & 429 & $158(36,8 \%)$ & 417 & $131(31,4 \%)$ \\
AB & 57 & - & 43 & - \\
\hline Gesamt & 1123 & $344(30,6 \%)$ & 1066 & $268(25,1 \%)$ \\
\hline
\end{tabular}

Tab. 6: Häufigkeit der Fehlbestimmungen des Rhesusfaktors bei 1590 untersuchten Neugeborenenblutproben bezogen auf die Ursache der Fehlbestimmung und die Herkunft der Blutproben. (NSB = Nabelschnurblut, $V B=$ Venenblut)

\begin{tabular}{|c|c|c|c|}
\hline & Gesamt & NSB & VB \\
\hline Untersuchte Blutproben & 1590 & 1028 & 562 \\
\hline $\begin{array}{l}\text { Ursache: } \\
\text { Schwaches Antigen, } \\
\text { negative Reaktion }\end{array}$ & 5 & 2 & 3 \\
\hline $\begin{array}{c}\text { Sćchwaches Antigen, } \\
\text { Fehlinterpretation }\end{array}$ & 3 & 2 & 1 \\
\hline Unspezif. Reaktion & 4 & 2 & 2 \\
\hline Gesamt & $\begin{array}{c}12 \\
(0,8 \%)\end{array}$ & $\begin{array}{c}6 \\
(0,6 \%)\end{array}$ & $\begin{array}{c}6 \\
(1,1 \%)\end{array}$ \\
\hline
\end{tabular}




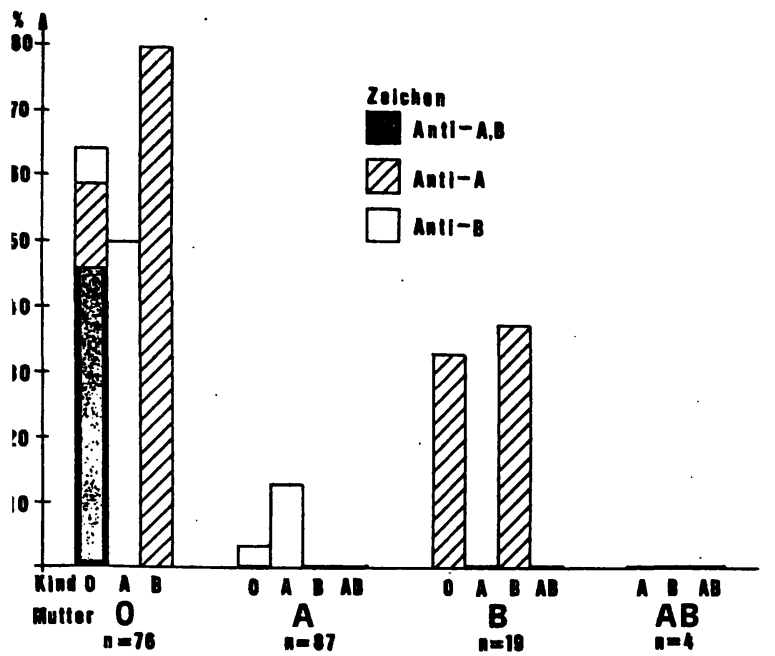

4bb. 1: Zusammenhang zwischen der mütterlichen Blutgruppe Ind der Häufigkeit "regulärer" agglutinierender AB-Antikörper 'Kochsalztest) im Neugeborenenblut

;chwache Reaktion protokolliert, aber als Rh-negativ ehlbefundet. 2 dieser 8 Neugeborenen waren bei der Jeburt unreif.

- 4mal führten deutliche, aber unspezifische Agglutinaionen zur Diagnose „Rh-positiv”.

- Die Frequenz der Fehlbestimmungen lag mit Venenblut iöher als mit Nabelschnurblut (1,06\% gegenüber $0,58 \%$ ), dies ergab jedoch im Chiquadrat-Test keinen signifikanen Unterschied ( $p>0,10)$.

\section{Diskussion}

3ei den beobachteten ABO-Fehlbestimmungen führten n der Mehrzahl der Fälle (6 von 11) unspezifische Reakionen zur Diagnose falscher Antigene. Für die FehlerfinJung im Rahmen der „langen Blutgruppe“ war v. a. die jrößere Spezifität der Antigenaustestung durch Vorwa;chen und Resuspendieren der Patientenerythrozyten sovie $2 \mathrm{mal}$ die Serumgegenprobe wesentlich. In 5 von I1 Fällen war die Fehlbestimmung durch mangelhafte Antigenausprägung auf den kindlichen Erythrozyten (imner Blutgruppenmerkmal A) bedingt. Diese Beobachung deckt sich mit den Ergebnissen von Kusnierz und -eskiewicz (7), welche eine deutlich verminderte Reaktiität von Nabelschnurerythrozyten im Vergleich zu Erwachsenenerythrozyten mit Anti-A-Testserum fanden. Zu dem Ergebnis, daß das A-Antigen bei Neugeborenen roch nicht die volle Ausprägung wie bei Erwachsenen zrfahren hat, kamen auch Economidou und Mit. (8), Grundbacher (9) sowie Constantoulakis (10). Die Rate Jer ABO-Fehlbestimmungen anhand der alleinigen Antijenaustestung war zwar mit Venenblut signifikant niedrijer als mit Nabelschnurblut, bei Beachtung der nachfoljenden Empfehlungen kann Nabelschnurblut jedoch zbenso gut wie Venenblut verwendet werden.

n über $32 \%$ aller untersuchten NeugeborenenblutgrupJen konnte die Blutgruppe allein anhand der im Kochsalzest gefundenen Isoagglutinine bestimmt werden. Nach Race und Sanger (11) sollen die im Nabelschnurblut geundenen Anti-A- und Anti-B-Antikörper passiv von der Mutter auf den Feten übertragen werden. Jedoch konn:en schon Toivanen und Hirvonen (4) in 8 von 44 Neugejorenenseren Anti-A-Antikörper bei Blutgruppe $A$ der
Mutter bzw. Anti-B-Antikörper bei Blutgruppe B nachweisen. Infolgedessen müssen Feten schon zur Synthese eigener AB-Antikörper befähigt sein. Vom 3. bis 6. Lebensmonat an ist dies die Regel (12). Auch anhand unserer Befunde dürften die AB-Antikörper im Neugeborenenblut mit wenigen Ausnahmen von der Mutter stammen, da im kindlichen Blut fast immer nur Antikörper nachgewiesen wurden, die im mütterlichen Blut vorkamen (Abb. 1).

Allerdings ist deren Nachweisbarkeit im kindlichen Blut mit dem Kochsalztest fast ausschließlich auf solche ABAntikörper beschränkt, die für die kindlichen Antigene kompatibel sind. Högman (13) konnte die Blutgruppenantigene $A$ und $B$ schon auf fetalen Zellen verschiedener Gewebe wie Niere, Leber, Milz, Lunge, Herz und Haut nachweisen und Friedhoff und Kuhns (14) fanden die Blutgruppenantigene $A, B$ und $H$ auf menschlichen Amnionzellen. Es dürfte damit ziemlich sicher sein, daß die AB-Antikörper im Neugeborenenblut weitgehend von der Mutter stammen, die für das Kind inkompatiblen ABAntikörper im Serum des Neugeborenen jedoch nicht auftauchen, da sie vorher von den korrespondierenden Blutgruppenantigenen der verschiedenen Gewebe des Feten neutralisiert werden. Im Endeffekt finden sich daher fast nur die für die Blutgruppenantigene kompatiblen $A B$ Antikörper im Neugeborenenblut.

Wir wiesen die kompatiblen AB-Antikörper signifikant häufiger in Nabelschnur- als in Venenblut nach. Bezogen auf die jeweilige Blutgruppe des Neugeborenen konnte jedoch überraschenderweise nur bei der Blutgruppe $A$ in dieser Hinsicht eine Signifikanz errechnet werden. Eine Erklärung für diese Beobachtung ist uns nicht möglich. Anti-B-Antikörper traten bei der Blutgruppe 0 in 12,3\% der Fälle alleine auf. Dieser Befund ist eher störend, da er die Fehlinterpretation zugunsten eines schwachen A-Antigens zur Folge haben kann. Dagegen wirkt sich der alleinige Nachweis von Anti-A-Antikörpern (6,6\% der Fälle) stützend auf die Diagnose der Blutgruppe 0 aus, da das Antigen B nur äußerst selten abgeschwächt vorkommt.

Unter den Fehlbestimmungen des Rh-Faktors im Rahmen der "'kurzen Blutgruppe“ führten unspezifische Reaktionen nur in 4 von 12 Fällen zur Fehlannahme von Rhpositiv. Doppelt so häufig wurde der Rhesusfaktor fälschlich negativ bestimmt; hier konnte kein Zusammenhang mit kindlicher Unreife festgestellt werden. Nach Angaben von Race und Sanger (15) sollen die Rhesusmerkmale im Gegensatz zu den ABO-Antigenen schon bei Geburt voll ausgeprägt sein. Chown (16) und Speiser (17) konnten sogar schon bei Feten im Gestationsalter von 4 bis 6 Wochen die Rhesusmerkmale nachweisen. Die von uns gefundene Frequenz von $0,5 \%$ falsch-negativ bestimmter Rhesusfaktoren entspricht nach Mollison (18) der Häufigkeit von $D^{u}$. Im Hinblick auf die postpartal durchzuführende Rhesusprophylaxe sind jedoch falschnegative Rhesusbestimmungen von erheblicher forensischer Bedeutung. Neben den von uns genannten methodischen Möglichkeiten könnte die Rate der Rh-FaktorFehlbestimmungen durch die Verwendung spezifischerer Antiseren, z. B. monoklonaler Antiseren, gesenkt werden. Entsprechende Antiseren für die Rhesusbestimmung sind jedoch noch nicht verfügbar. Für die Fehlerfindung im Rahmen der von uns durchgeführten "langen Blutgruppenbestimmung" waren höhere Empfindlichkeit und gröBere Spezifität sowie Austestung der Rhesusformel einschließlich „Du-Test” bei Fällen mit Abschwächung bzw. Negativität gegenüber Anti-D und Positivität gegenüber Anti-CDE im direkten Testverfahren (sog. „Rh-Zwitter”) 
entscheidend. Es ergab sich kein signifikanter Unter-. schied in der Häufigkeit der Rhesusfaktorfehlbestimmungen bei Verwendung von Nabelschnurblut gegenüber Venenblut.

\section{Methodische Empfehlungen}

Blutgruppenfehlbestimmungen bei Neugeborenen können durch folgende Testbedingungen reduziert werden:

- Waschen und Resuspendieren der Erythrozyten in physiologischer Kochsalzlösung.

- Verwendung von zwei Testserensätzen, Anwendung von monoklonalen Antikörpern. Monoklonale AB-Antikörper eignen sich insbesondere als Zusatz zu den polyklonalen Testseren, um schwache AB-Antigene besser nachzuweisen (19).

- Längere Inkubationszeiten

Die ABO-Bestimmung soll endgültig erst nach 2030 min befundet werden. Dennoch kann für evtl. eilig bereitzustellende Blutkonserven schon der 5-min-Befund herangezogen werden. Die Inkubationsdauer bei der Rhesusbestimmung sollte im Wärmeblock/Wasserbad mindestens 15 min und im Brutschrank 30 min betragen.

- Definierte Inkubationstemperatur

(AB0: Raumtemperatur $20-25^{\circ} \mathrm{C}$ ), Rhesus: $37^{\circ} \mathrm{C}$.)

- Bestimmung der Rhesusformel mit agglutinierenden und inkompletten Antiseren.

- Indirekter Coombstest mit inkomplettem Anti-D bei Ccdee, ccdEe, CcdEe.

- Serumgegenprobe im Kochsalztest bei der ABO-Bestimmung.

Befunde von Neugeborenenblutgruppen sollten nicht in Impf- und Blutgruppenausweise aufgenommen werden. Im Zusammenhang mit Bluttransfusionen ist die Blutgruppe bis zur vollen Ausprägung der Antigene und $A B$ Antikörper regelmäßig zu wiederholen (bis Ablauf des 1. Lebensjahres).

\section{Schriftum:}

1. THOMAIDIS, T., FOUSKARIS, G., MATSONIOTIS, N.: Isohemagglutinin activity in the first doy of life. Amer. J. Dis. Child. 113, 654 (1967).

2. ChatTORAY, A., GILBEAT, R., JOSEPHSON, A. M.: Serological demonstration of fotal production of blood group isoantibodies. Vox Sang. 14, 289 (1968).

3. THOMAIDIS. T. AGATHOPOULOS, A., MATSONIOTIS, N.: Natural isohemogglutinin production by the fotus. J. Pediatr. 74, 39 (1969).

4. TOIVANEN, P., HIRVONEN, T.: Iso- and heteroagglutinins in human fetal and neonatal sera. Scand. J. Haematol. 6, 42 (1969).

5. FONG, S. W., QUAQUNDAK, B. Y., TAYLOR, W. F.: Developmental patterns of $A B O$ lsoagglutinins in normal children correlated with the effects of age, sex, and maternal isoagglutinins. Transfusion 14, 351 (1974).

6. BUNDESÄRZTEKAMMER: Richtlinien zUr Blutgruppenbestimmung und Bluttransfusion. Dtsch. Arzteblatt 5, 277 (1979).

7. KUSNIERZ, G., LESKIEWICZ, A.: Studies on the ABH component of human newborn erythrocytes. Asch. Immunol. Ther. Exp. 19, 635 (1971).

8. ECONOMIDOU, J., HUGHES.JONES, N. C., GARDNER, B.: Quantitative measurements concerning $A$ and $B$ antigen sites. Vox Sang. 12, 328 (1967).

9. GRUNDBACHER, F. J.: Changes in the Human $A$ antigen of erythrocytes with the individual's age. Nature 204, 192 (1964).

10. CONSTANTOULAKIS, M., KAY. H. E. M.: A and $B$ antigens of the human fetal erythrocyte. Br. J. Haematol. 8, 57 (1962).

11. RACE, R. R., SANGER, R.: Blood groups in man, S. 45. 6th edition (1975).

12. MORVILLE, P.: Investigation on iso-haemagglutination in mothers and newborn children. Acta Pathol. Microbiol. Scand 6, 39 (1929).

13. HOGMAN, C. F.: Blood group antigens $A$ and $B$ determined by means of mixed aggiutination on cultured cells of human fetal kidney, liver, spleen, lung, heart and skin. Vox Sang 4, 319 (1959).

14. FRIEDHOFF, F., KUHNS, W. J.: Detection and characterization of blood group antigens on untransformed human amnion cells. Transfusion 8, 244 (1968).

15. RACE \& SANGER, ders. S. 236.

16. CHOWN, B.: On a search for thesus antibodies in very young foetuses. Arch. Dis. Childh. 30, 232 (1955).

17. SPEISER, P.: Über die bisher jüngste menschliche Frucht $(27 \mathrm{~mm}, 2,2 \mathrm{~g})$ an der bereits die Erbmerkmale $A_{1}, M, N, s, F y\left(a^{+}\right), C, C, D, E, e, J k\left(a^{+}\right.$?) im Blut festgestellt werden konnten. Wien. Klin. Wschr. 71, 549 (1959).

18. MOLLISON, P. L.: Blood transfusion in clinical medicine. 7th edition, S. 338 (1983)

19. MESSETER, L., BRODIN, T., CHESTER, M. A., LOW, B., LUNDBLAD, A.: Mouse monoclonal antibodies with anti-A, anti-B and anti-A,B specifities; some superior to human polyclonal ABO reagents. Vox Sang. 46, 185 (1984).

\section{Anschrift der Verfasser:}

Abt. für Transfusionsmedizin und Gerinnungsphysiologie

Universitätsklinikum

Philipps-Universität

3550 Marburg 
TOSY = das modulare elektiv=Analysen-Systemo indmitcmarkjerungskarten rogrammiert, dieders efandelnde Arztdirekt bet Eventersuchung ausfitilt. togrammieren fim Labor Itfalle. Daswachtithre rbeitsicher lochdas MOSSY bietretauch 3linvestifion Sicherheits icherthefibelm Kauf denn 3 istpreiswertund spars im. Sicherheitsinden rgebnissen denn:Markies Ingskarten programmieren Shlerfreib Sicherheitsom auerbetriebs denn MTOSY? Beinfach zubedienenund Ijverlässiog

Eshalb garantieren wat fur des MOSY zweil thre ostenlose Wartung 3oBrunglLangeGinbH errefre Medizin of fosweg (10 F1000 Berfin 37 nddinithamburg, Erankfurt ndilionchens

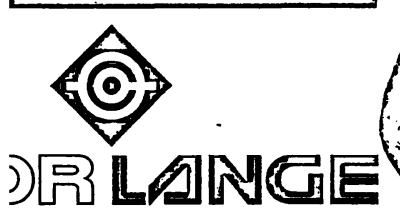

Ganz einfach. MOSY arbeitet direkt naeh Vorschrift vom Apztito

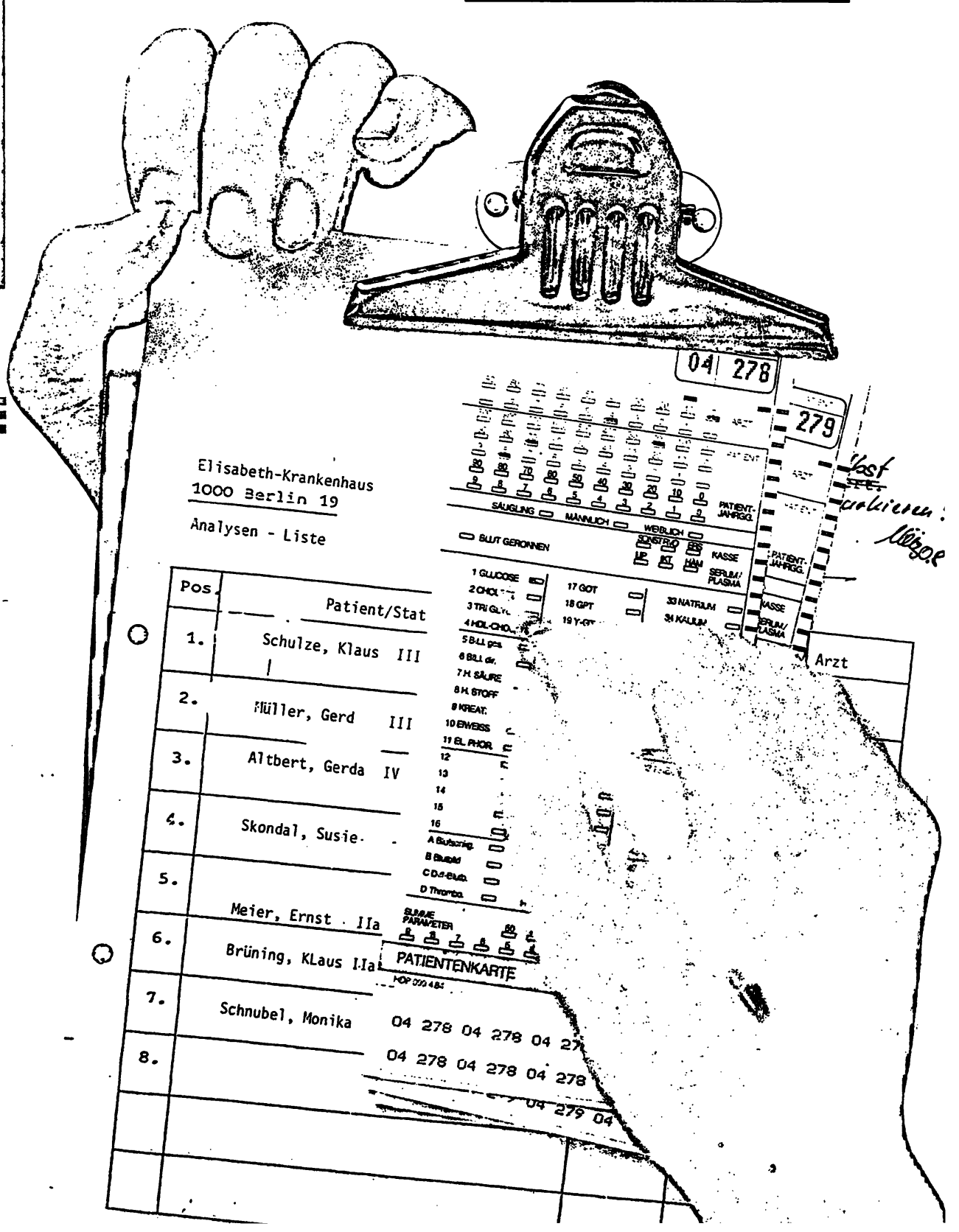



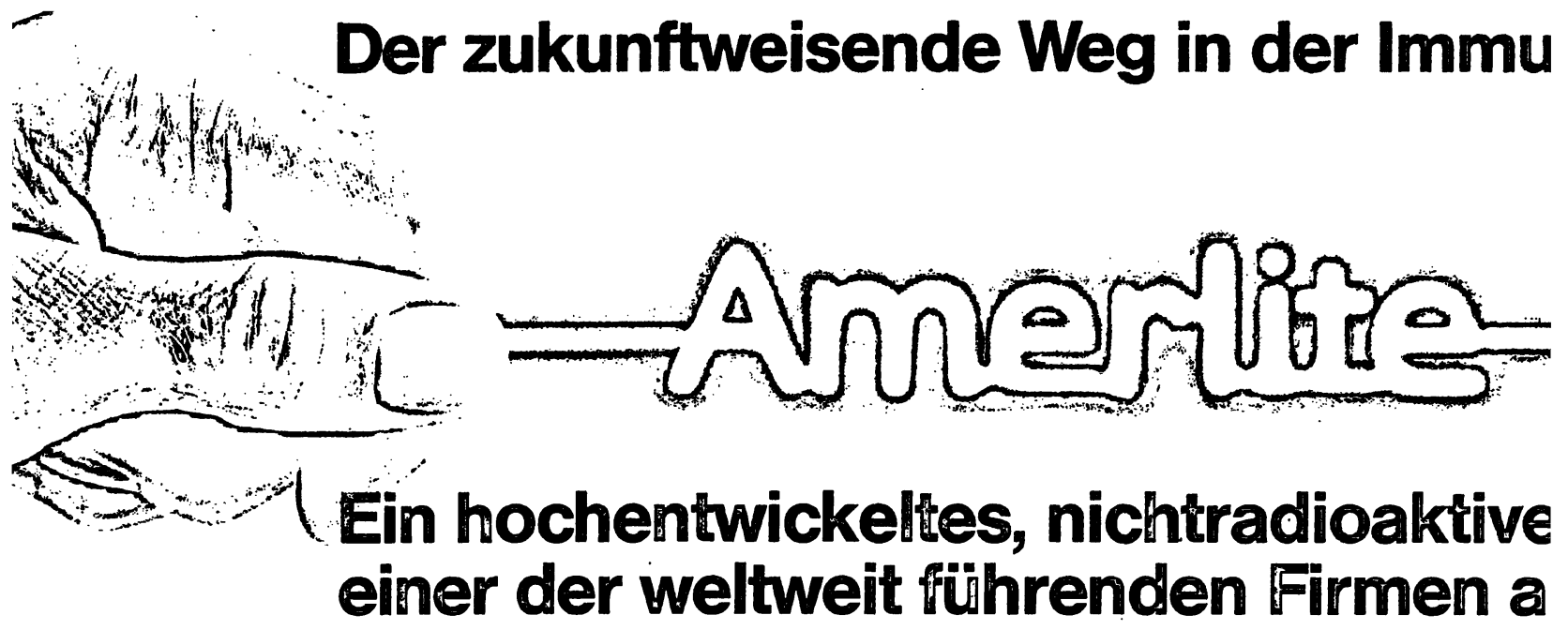

\section{DAS SYSTEM}

mit den neuesten technologischen Entwicklungen

- signalverstärkte Lumineszenz

- beschichtete Mikroröhrchen als Träger

- immunometrische Assays

- monoklonale Antikörper

- speziell entwickelte Geräte

Amerlite bietet zuverlässigere Resultate als bisherige Systeme, größere Flexibilität bei der Durchführung und ein hohes $\mathrm{MaB}$ an Anwenderfreundlichkeit.

\section{UNIVERSELL EINSETZBAR}

Amerlite ist im gesamten Analytenspektrum einsetzbar, denn es ermöglicht die Bestimmung von großen und kleinen Molekülen im Gegensatz zu herkömmlichen Enzym- und Fluoreszenzimmunoassays.

Amerlite kann in jedem Labor eingesetzt werden. Die Messung erfolgt ohne Einsatz von Radioaktivität; eine Umgangsgenehmigung für den Einsatz radioaktiver Stoffe entfällt; Entsorgungskosten fallen nicht mehr an.

\section{DIE QUALITÄT}

Amerlite mit signalverstärkter Lumineszenz weist wesentliche Vorteile gegenüber anderen nichtradioaktiven Immunoassay-Techniken auf und bringt Resultate von hoher Qualität durch:

- Sensitivität

- Richtigkeit und Präzision der Resultate

- Breite des Meßbereichs

- Zuverlässigkeit

\section{DIE ANALYTEN}

Zur Zeit verfügbare Amerlite Assays:

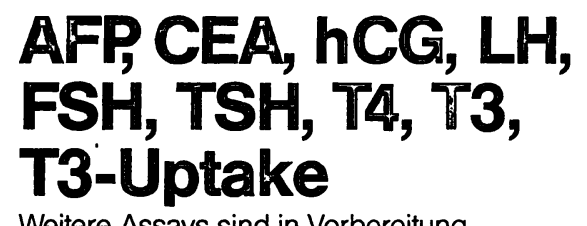

Weitere Assays sind in Vorbereitung.

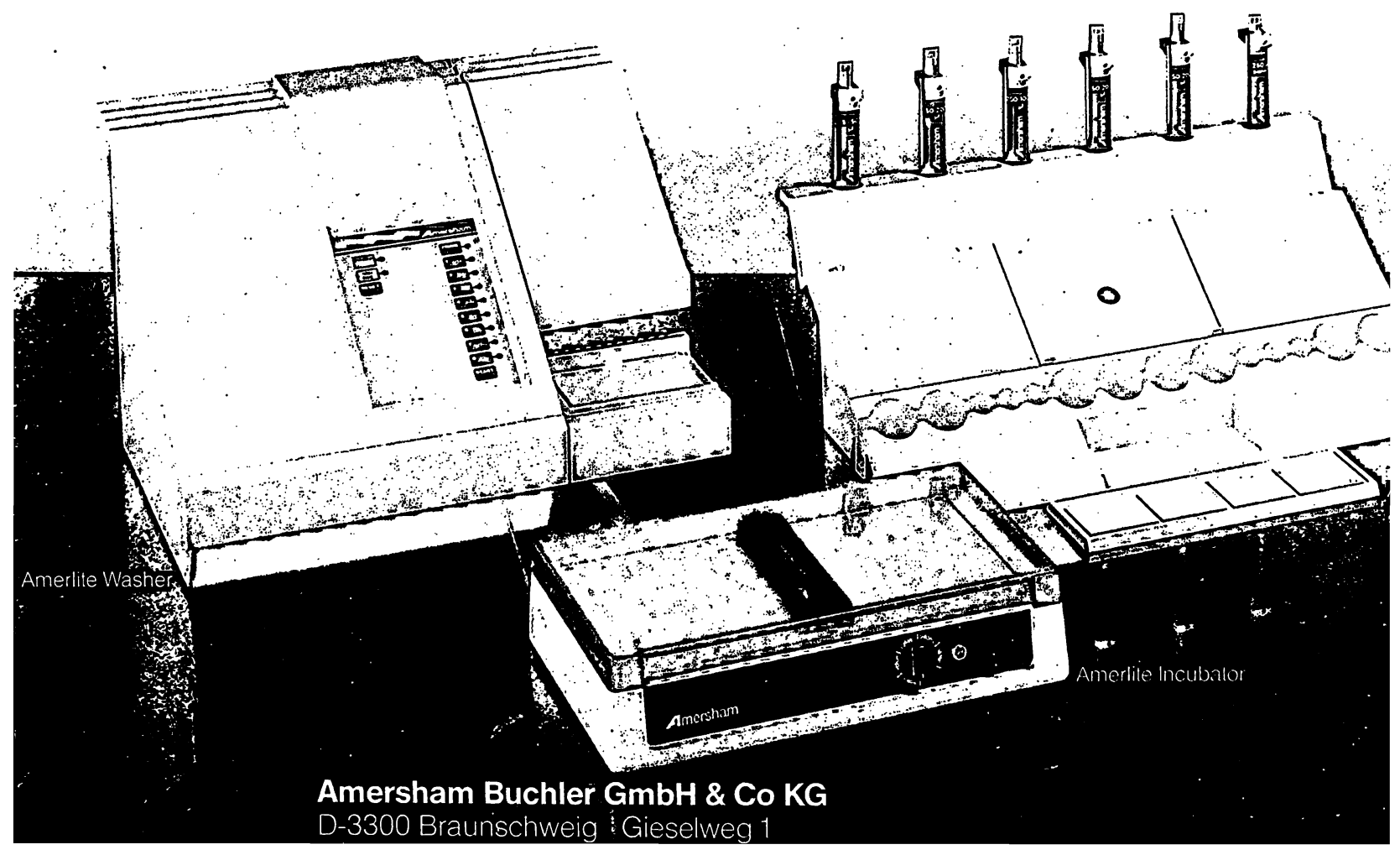




\section{y-Technologie}

\section{Im von Amersham International, Gebiet der Immunoassay-Technologie}
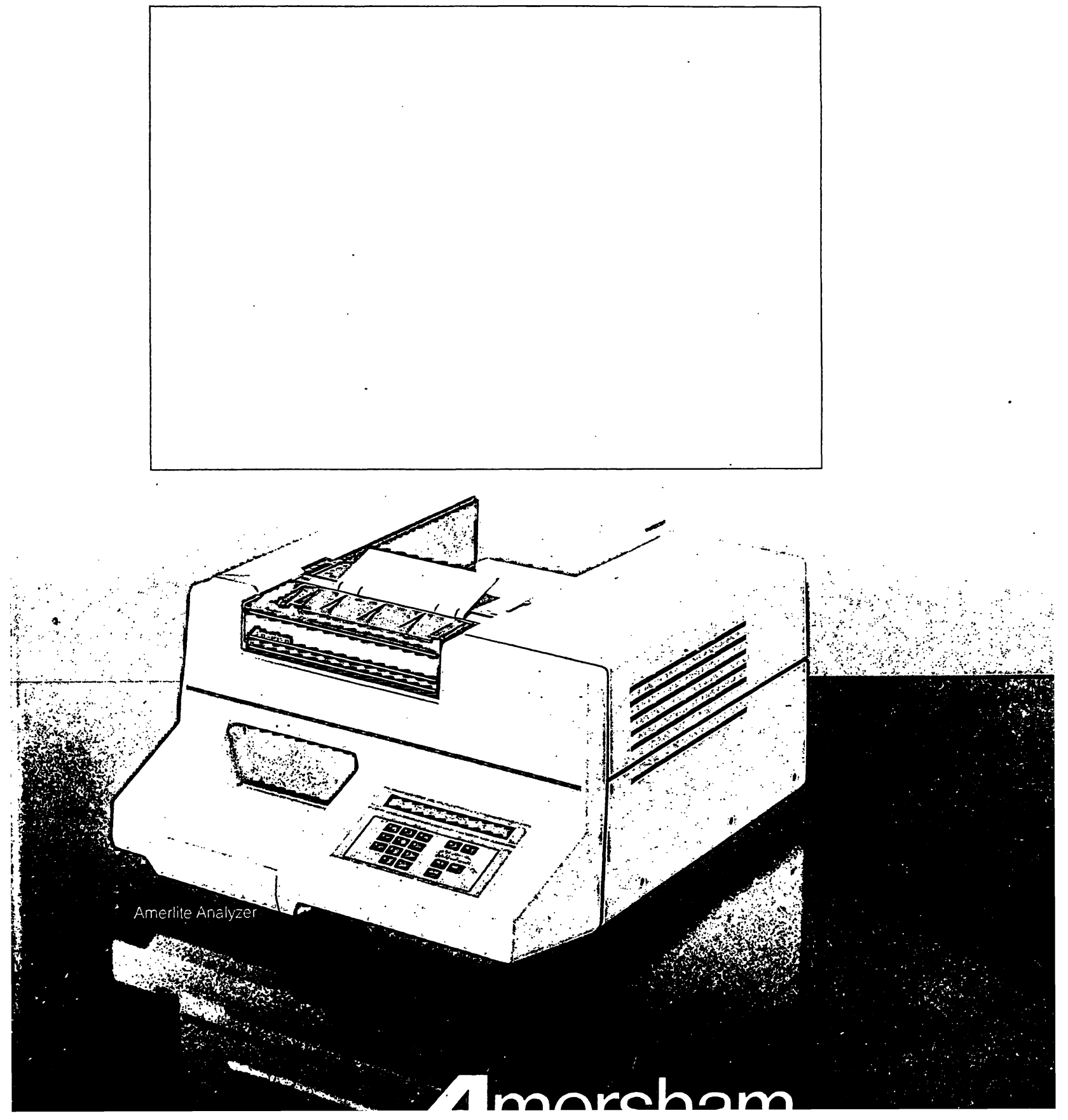


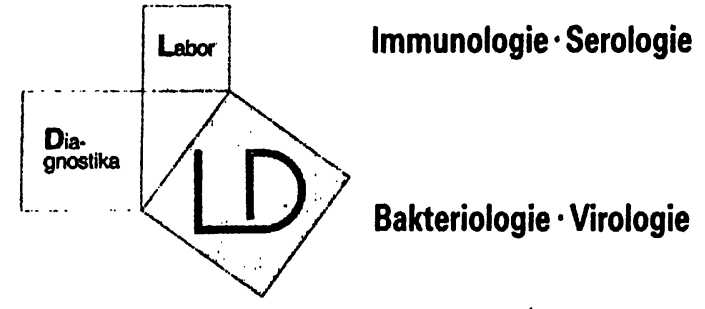

\section{LD Sensifluid für die}

Resistenzbestimmung im Agardilutionsverfahren

sicher - preiswert - flexibel - schnell

LD Sensifluid ist ein für das Routinelabor konfektioniertes System.

- Lyophilisierte Antibiotika in den vom Anwender gewünschten Konzentrationen.

- Durch verschiedene Packungsgrößen individuell auf jedes Labor adaptierbar.

Unsere Mikrobiologen helfen Ihnen beim Umsetzen von der Theorie in die Praxis und der Erstellung kostenoptimierter Antibiogramme.

Lassen Sie sich überzeugen:

Bessere Mikrobiologie für weniger Geld

\section{LD-Lieferprogramm}

Immunfluoreszenzprodukte von CSI . Elisas - Rheumadiagnostik - Toxoplasmosediagnostik - Luesdiagnostik · IgMDiagnostik - Diagnostische Antiseren TB-Diagnostik - Transportmedien - Fertigplatten - Mykologie - Blutkulturen Supplemente - Testringe - Differenzierungsblättchen.

NEU: CLIN-ENA Hämagglutinationstest zur Bestimmung der Antikörper gegen extrahierbare nukleäre Antigene (Sm/RNP-Differenzierung)

Bitte fordern Sie unsere Gesamtproduktliste an.

L-D Labor Diagnostika GmbH Industriestraße 12 D-4284 Heiden/Westf. Telefon (0 28 67) 8083

Tx 813423 Idlab d
Warum

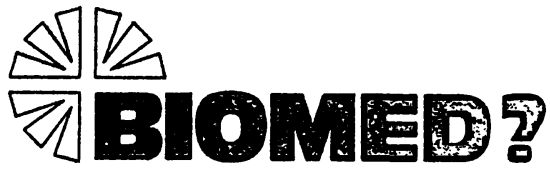

Weil bei BHOMED

O Qualitat

vor Markt-

beherrschung,

O Fexiloilfect

vor Größe und

$\bigcirc$ entwicklunng

vorWerbung steht.

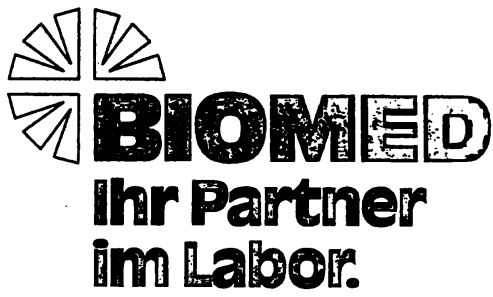

IhrVorteil bei
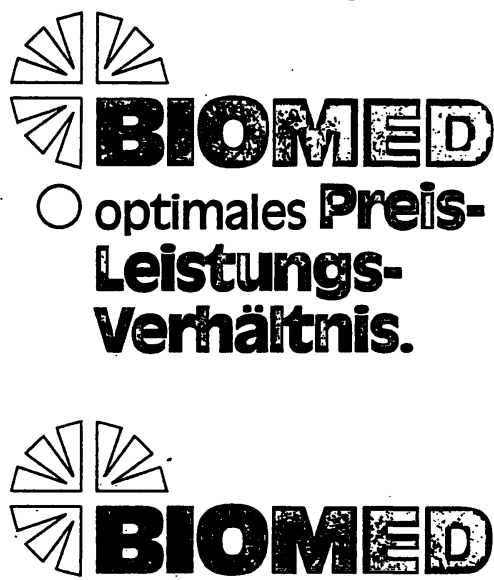

Einfacher. Sicherer. Einfach sicher.
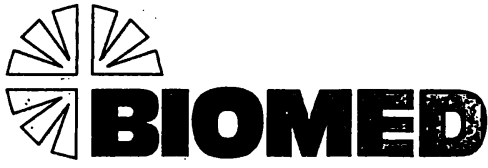

Labordiagnostik $\mathrm{GmbH}$

Bruckmannring 28

8042 Oberschleißheim

bei München

Service-Telefon:

(089) 3151619 